rev.relac.int.estrateg.segur.7(1):75-97,2012

\title{
ELEMENTOS DE REFLEXIÓN PARA MEJORAR LA PRÁCTICA DE LA COOPERACIÓN OFICIAL DESCENTRALIZADA EN LOS MUNICIPIOS COLOMBIANOS*
}

\author{
Denisse Amara Grandas Estepa**
}

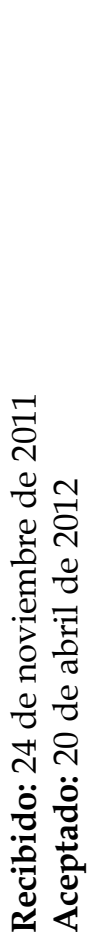

\section{RESUMEN}

La Cooperación oficial descentralizada (COD), es una modalidad de cooperación para el desarrollo cuyos actores principales son los gobiernos locales y regionales.

Este artículo describe las características más importantes de las relaciones de COD desde un "modelo vertical" tradicionalista y un "modelo horizontal" que pretende avanzar hacia relaciones soportadas en la reciprocidad entre socios. Bajo este contexto, se analiza el comportamiento de la cooperación de las Comunidades autónomas (CC AA) españolas con mayor presencia en el país en el periodo 1995-2007 y se plantean algunos elementos de reflexión para avanzar de una

* El articulo es producto del proyecto de investigación construcción de un modelo horizontal de cooperación descentralizada para municipios en Colombia. Experiencia Piloto, Caldono, Cauca. Identificado con el número 323-06-10, financiado por la UJTL y hace parte del Grupo de investigación en política y relaciones internacionales del Programa de relaciones internacionales. Soy la investigadora principal. El proyecto culmina en septiembre de 2012.

** Internacionalista de Universidad Jorge Tadeo Lozano con Maestría en Desarrollo y Ayuda Internacional de la Universidad Complutense de Madrid, España. Docente asociada e investigadora del Programa de Relaciones Internacionales de la Universidad Jorge Tadeo Lozano. denisse.grandas@utadeo.edu.co 
visión tradicionalista hacia formas más equilibradas y reciprocas que mejoren la calidad y la eficacia de la COD y que beneficien los procesos de desarrollo local en Colombia.

Palabras Clave: Cooperación oficial descentralizada, modelo horizontal, modelo vertical, España, Colombia.

\section{ABSTRACT}

Decentralized Official Cooperation (COD) is a form of collaboration for development, whose main actors are the local and regional governments.

This article describes the most important characteristics of the relations of COD from a traditional "vertical model" and a "horizontal model" that aims to proceed towards relations supported by reciprocity between partners. Under this context, the performance of the cooperation of the Spanish Autonomous Communities (CCAA) with stronger presence in the country during the period 1995-2007 is analyzed, and some elements of reflection are presented to advance from a traditional vision towards more balanced and reciprocal facets that improve the quality and effectiveness of COD and that benefit the processes of local development in Colombia.

Key Words: Decentralized official cooperation, horizontal model, vertical model, Spain, Colombia.

\section{ELEMENTOS DE REFLEX̃̃o PARA A MELHORIA DA PRÁTICA DE COOPERAÇÃO OFICIAL DESCENTRALIZADA NOS MUNICÍPIOS COLOMBIANOS}

\section{RESUMO}

A cooperação oficial descentralizada (COD) é uma forma de cooperação para o desenvolvimento, cujos principais atores são os governos locais e regionais.

Este artigo descreve as características mais importantes das relações de COD desde um "modelo vertical" tradicionalista e um "modelo horizontal", que procura avançar para relações apoiadas na reciprocidade entre os parceiros. Sob este contexto, analisa-se o comportamento da cooperação entre as Comunidades autônomas (CC AA) espanholas com maior presença no país no período de 1995-2007 e são propostos alguns elementos de reflexão para avançar de uma visão tradicionalista para formas mais equilibradas e recíprocas que melhorem a qualidade e a eficiência da COD e que beneficiem os processos de desenvolvimento local na Colômbia.

Palavras Chave: Cooperação oficial descentralizada, modelo horizontal, modelo vertical, Espanha, Colômbia. 


\section{INTRODUCCIÓN}

Con la revalorización de la dimensión territorial-local, los gobiernos territoriales ${ }^{1}$ han emprendido relaciones internacionales y han buscado mecanismos de internacionalización a través de procesos de cooperación e interlocución con actores nacionales, regionales y mundiales.

Los intercambios y los procesos de cooperación entre este tipo de gobiernos "no centrales" han constituido hoy una verdadera "diplomacia de ciudades" (Zapata, 2007) o "paradiplomacia", con el propósito de fortalecerse en lo institucional, económico, cultural, político y social.

En este escenario, se fortalece la cooperación oficial descentralizada (COD) como una nueva modalidad en el sistema de cooperación para el desarrollo, cuyos actores principales son los gobiernos municipales y regionales, quienes por decisión propia se ven estimulados a establecer procesos de acción exterior a través de redes internacionales, hermanamientos, ejecución de proyectos, y desplegar sus propias capacidades de proyectar y llevar a cabo iniciativas de desarrollo.

España se ha convertido en uno de los grandes impulsores de este tipo de cooperación gracias a la presencia de varias de sus comunidades autónomas (CC AA) y de las entidades locales, en los países en desarrollo. En Colombia, la ha promovido especialmente desde las CC AA de Madrid, País Vasco, Cataluña, Valencia, Navarra, Asturias e Islas Baleares.

Sin embargo, para algunos municipios del país la COD es desconocida y para otros, con mayor capacidad de gestión, ha sido utilizada como un mecanismo de internacionalización y de financiación de proyectos de desarrollo.

Aun así, en el país es una cooperación que no ha sido lo suficientemente explorada y aprovechada como consecuencia de la falta de desarrollo de capacidades en los gobiernos territoriales, la ausencia de mecanismos de información consolidados, la dispersión, la poca coordinación y articulación de las iniciativas en estructuras y estrategias planificadas que beneficien los procesos de desarrollo local.

Este artículo presenta los elementos más importantes de la COD y describe, de manera general, dos modelos que reflejan las diferentes prácticas y modalidades que se han evidenciado en este tipo de ayuda al desarrollo. Frente a estos dos enfoques, se identifican las características más importantes de la experiencia de las CC AA en Colombia y se plantean algunos elementos de reflexión para mejorar los procesos de COD en el país, con un énfasis especial en relaciones

1. Cuando se habla de gobiernos territoriales, se hace alusión a las administraciones municipales e incluso regionales de carácter descentralizado. 
horizontales, equilibradas, durables y sostenibles que sobrepasen las tradicionales relaciones de ayuda a proyectos específicos.

El artículo es producto del proyecto de investigación "Construcción de un modelo horizontal de cooperación descentralizada para Colombia. Experiencia piloto: Municipio de Caldono, Cauca", el cual hace parte del Grupo de investigación en política y relaciones internacionales del Programa de Relaciones Internacionales de la Universidad Jorge Tadeo Lozano.

\section{LAS RELACIONES INTERNACIONALES DE LOS MUNICIPIOS: BREVE CONTEXTUA- LIZACIÓN}

Desde hace varios años se ha venido evidenciado la presencia cada vez más creciente de las entidades territoriales en el escenario internacional. En esta nueva dimensión se integra lo local y lo global, en la cual se encuentra una nueva oportunidad para el desarrollo por medio de labores relacionadas con la proyección y la promoción internacional del ente local, la búsqueda de visibilidad en el extranjero, la atracción de inversiones, el comercio y el turismo (Zapata, 2007).

Implementada en el orden local, la internacionalización municipal entonces, es esa participación activa del territorio en el sistema internacional, que busca acceder a recursos, intercambiar experiencias y conformar redes de cooperación e integración económica, con el fin de encontrar consensos y soluciones alrededor de temáticas comunes (FCM, 2009).

Las relaciones internacionales de los gobiernos territoriales también han sido enmarcadas en lo que comúnmente se conoce como paradiplomacia, en la cual los procesos de internacionalización de los gobiernos territoriales se fundamentan en un ejercicio paradiplomático cuyo fin último es la promoción del desarrollo local.

Este término aparece en los años ochenta, periodo en el cual el debate se centró en los cambios que se empezaron a evidenciar en los estados federales Norteamericanos, en especial, en las relaciones de los gobiernos federales y los estados federados en el campo de la política exterior. (Aguirre, 2000).

Sin embargo, a lo largo de los años este neologismo ha sido motivo de una variedad de discusiones y de aproximaciones a nuevos términos para describir, desde diferentes perspectivas, la dinámica de las relaciones internacionales de los gobiernos no centrales. Así entonces, en los años ochenta se propuso el término microdiplomacia para referenciar el concepto y la práctica de las actividades internacionales y la autonomía de la política territorial (Duchacek, 1984). Otros escritores utilizaron términos como "baja política" relacionado con los intercambios económicos, sociales y culturales de los municipios, en contraste con el significado tradicional de la política exterior o "alta política". (Duchacek, 1984). 
Posterior al término microdiplomacia surge el neologismo paradiplomacia el cual se le atribuye a Panayotis Soldatos, para describir la actividad internacional de los gobiernos no centrales. (Aguirre, 2000). A partir de entonces, se proponen una variedad de términos como protodiplomacia, relacionado con las iniciativas de los gobiernos no centrales en el extranjero y con los mensajes separatistas de algunas regiones, diplomacia multinivel o diplomacia catalítica propuesto por Brian Hocking, en el que se plantea que la diplomacia no es atributo exclusivo de los Estados y se avanza hacia una visión que reconoce a los gobiernos no centrales como actores internacionales tan confusos como el Estado, los cuales deben ocuparse de aspectos relacionados con la industria, el comercio, la educación, la cultura, la política social, la vivienda las finanzas y la seguridad. (Aguirre, 2000)2.

Si bien, la acción exterior de los municipios ha sido motivo de una variedad de discusiones y de aproximaciones a nuevos términos, la comprensión de la dimensión internacional se ha convertido en una alternativa válida para los municipios que buscan dar solución a sus problemas de desarrollo así como para obtener apoyo a sus iniciativas de gestión, como respuesta a los nuevos desafíos de la globalización.

Sin duda alguna las relaciones internacionales desde los municipios han sido producto del mismo proceso de globalización en el que se evidencia la necesidad de reorientar el accionar de las unidades políticas menores a las del estado-nación y generar el desarrollo de nuevas capacidades en el nivel local.

En este mismo sentido, el ámbito local ha comenzado a tener una importancia política como consecuencia de los procesos de democratización y reformas del Estado, descentralización y desconcentración administrativa. La descentralización ha fortalecido las estructuras territoriales del orden subnacional ampliando sus capacidades y ámbitos de actuación, y otorgándoles un mayor grado de autonomía, de control de los recursos y de mayores responsabilidades y competencias, de manera que, pueda potenciarse la interacción y acercamiento entre el Estado y sus comunidades. A su vez, la descentralización ha sido una estrategia para responder a las exigencias de la globalización y un instrumento inherente a la democracia participativa. Se asume que en los procesos de descentralización se mejoran los niveles de eficacia del Estado y se amplía la base social, potenciando la gobernabilidad y la participación ciudadana.

De tal forma, los gobiernos territoriales buscan hoy activar respuestas a sus problemas de desarrollo y conseguir apoyo a sus iniciativas de gestión, a partir de nuevos modelos extensivos y comprensivos de la dimensión internacional. Así encuentran múltiples posibilidades de internacionalización a través de la generación de hermanamientos de ciudades, eventos para el fomen-

2. En la literatura académica se han propuesto otros conceptos para explicar las actividades internacionales de los gobiernos no centrales como paradiplomacia transfronteriza, regionalismo transfronterizo. 
to comercial o turístico, prestamos, presencia en foros internacionales (Díaz, 2008), atracción de inversión extranjera, cooperación descentralizada y participación en redes internacionales, cabildeo, lobby; mecanismos, todos ellos, a partir de los cuales, los gobiernos locales concurrirán en el nivel global, a la búsqueda de colaboración y coparticipación para el éxito de diversas iniciativas de gestión, al igual que para el desarrollo de planes y programas de desarrollo local.

\section{LA COOPERACIÓN OFICIAL DESCENTRALIZADA COMO UNA MODALIDAD POTEN- CIAL DE INTERNACIONALIZACIÓN MUNICIPAL}

Uno de los grandes instrumentos de internacionalización municipal viene estrechamente relacionado con la cooperación internacional. Así, los gobiernos territoriales han empezado a tener una presencia cada vez más activa y se han convertido en sujetos activos de las políticas de cooperación para el desarrollo. (Fernandez de Losada, 2010).

Esta dinámica hace parte de los procesos de cambio que se empezaron a evidenciar en el escenario de la cooperación a partir de los años noventa y que han sido enmarcados en lo que comúnmente se conoce como la "nueva arquitectura de la ayuda", la cual se caracteriza por el establecimiento de nuevos objetivos, actores, instrumentos y principios relacionados con la eficacia de la ayuda.

Frente a los objetivos, la comunidad internacional ha definido un nuevo consenso sobre el desarrollo para avanzar en el propósito de reducción de la pobreza. Así entonces, mediante la Declaración del Milenio y de los Objetivos de Desarrollo del Milenio (ODM) se ha conformado una agenda que compromete al conjunto de la comunidad internacional y que sin duda ha generado una transformación en las políticas de cooperación para el desarrollo ${ }^{3}$.

Por su parte, y ligado a los ODM, se han gestado una serie de propuestas reformadoras como consecuencia del reconocimiento y la necesidad de mejorar la eficacia de la ayuda (Alonso, 2010). La Declaración de Roma sobre Armonización (2003), la Declaración de Paris sobre eficacia de la ayuda (2005) y el Programa de Acción de Acrra (2008), que han dado lugar a la agenda de Paris, se constituyen entonces, en las referencias más importantes para comprender los nuevos compromisos entre donantes y receptores para avanzar en la eficacia de la ayuda.

Así mismo, se han incorporado al sistema de cooperación nuevos actores como las Organizaciones no Gubernamentales (ONG), las grandes fundaciones, los gobiernos territoriales y la participación de los países de renta media como oferentes de cooperación. Como consecuencia de

3. Es importante destacar la Conferencia sobre financiación del desarrollo de Monterrey en el año 2002, como un escenario importante en el que se buscaban acuerdos para el cumplimiento de los ODM y en el que los donantes evidenciaron la necesidad de mejorar la eficacia de la ayuda. 
esto, se empiezan a evidenciar nuevas modalidades de ayuda como la cooperación descentralizada (CD), la cooperación Sur-Sur y la cooperación triangular.

Así entonces, a partir de los noventa, con una concepción más moderna y funcional, se comienza a plantear la $\mathrm{CD}$ concebida como el conjunto de iniciativas de cooperación al desarrollo, que bajo el liderazgo de autoridades locales y/o la sociedad civil, procura estimular las capacidades de los actores de la base territorial y fomentar un desarrollo más participativo (Zapata, 2007).

La cooperación oficial descentralizada (COD), por su parte, se refiere exclusivamente a aqueIlas actuaciones llevadas a cabo desde los gobiernos territoriales, de manera directa o a través de la sociedad civil. En esta modalidad, los municipios en su nuevo rol deciden entran en contacto con otros municipios y con entidades otorgantes, con cierta autoridad para la negociación (Pérez, 2007).

El rol de los agentes, municipios, organizaciones de la sociedad civil, dirigentes empresariales y empleados, etc., que trabajen en la promoción del desarrollo resulta fundamental. Se trata de resaltar la importancia de la participación y movilización de los actores territoriales y de la necesidad de construir capital social para las iniciativas de desarrollo local (Alburquerque, 2004).

La COD implica entonces, una cooperación de una "colectividad territorial" dentro de un país o Estado con otra de otro país o Estado e incluye temas muy diversos presentados a escala regional y/o local. Su objetivo consiste en complementar los esfuerzos en ámbitos territoriales y sectoriales.

Se evidencia con esto, una cooperación que promueve el nexo entre las comunidades locales organizadas de los países en desarrollo y desarrollados mediante acuerdos de cooperación bilateral o multilateral, la participación en redes y el establecimiento de relaciones acordes con la realidad local.

Dentro de los beneficios más importantes de posicionamiento internacional de un municipio a través de la cooperación descentralizada están:

- La cercanía y participación de la sociedad civil en los procesos de desarrollo del municipio.

- Los vínculos directos entre homólogos que potencian las capacidades del municipio.

- La construcción de agendas a nivel internacional y de estrategias de marketing territorial.

- La autonomía respecto al conjunto de la cooperación al desarrollo, al desplegar capacidades para gestionar vínculos e intercambiar experiencias. 
- La coordinación y participación en redes de gobiernos locales y regionales.

- $\quad$ La posibilidad de potenciar el desarrollo local y fortalecer los procesos descentralizadores.

- El fortalecimiento del sistema de cooperación para el desarrollo a través de relaciones de carácter más horizontal que sobrepasan el tradicional asistencialismo y que permiten el intercambio de experiencias entre "socios".

- Potenciar no sólo su economía y comercio, sino los campos cultural, social y político.

- La generación de condiciones que permitan a los gobiernos locales operar eficientemente y reforzar los procesos de descentralización y la autonomía local (Fernández de Losada, 2007).

- La interacción en función de intereses y características comunes, estableciendo un contacto directo con actores mundiales de diversa índole.

- La participar en redes internacionales.

- $\quad$ Además de ayuda, la obtención de personalidad en el derecho internacional, posicionamiento en el exterior y construcción de una fuerza local (Rodríguez, 2005).

En este sentido, la cooperación descentralizada, se constituye en un buen instrumento para establecer relaciones, contribuir a una mayor autonomía y se convierte en una vía para potenciar los gobiernos territoriales como conductores del desarrollo local y la cohesión social.

\section{LA PRÁCTICA DE LA COD: EI MODELO VERTICAL Y HORIZONTAL}

Los movimientos solidarios y la movilización ciudadana de principios de los noventa que buscaban comprometer a los países ricos con los procesos de desarrollo generan un impacto fundamental en los gobiernos locales europeos, los cuales comienzan a destinar una parte de su presupuesto a acciones de solidaridad que se convierten en la premisa fundamental de la cooperación europea y le dan fuerza a la COD.

Es desde este momento donde se empiezan a identificar algunas características y prácticas frecuentes de COD vinculadas con la naturaleza de las relaciones y con el tipo de acciones que se ponen en marcha, que pueden ser sintetizadas en dos modelos de COD, tal como se indica en la figura No. 1. 


\section{La práctica de la COD: el modelo vertical y horizontal: características}

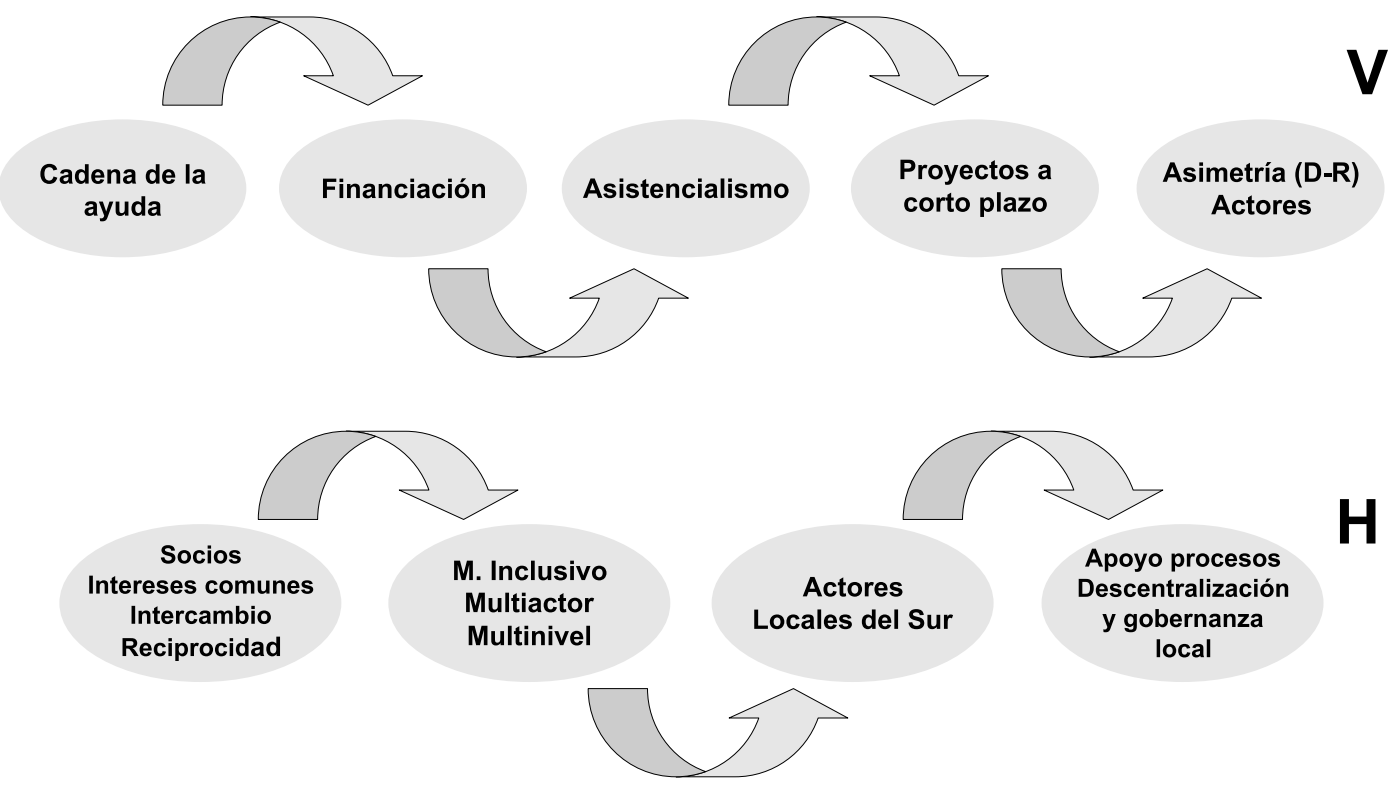

Figura No. 1. La práctica de la COD: el modelo vertical y horizontal

Fuente: Elaboración propia a partir de datos de Malé (2008) y Sanahuja y Martinez (2009)

El enfoque vertical se caracteriza por una práctica de COD tradicionalista de carácter asistencialista, donde los gobiernos territoriales del "Norte" actúan como financiadores y los actores del "Sur" (organizaciones de la sociedad civil y/o gobiernos territoriales), como receptores de cooperación (Sanz, 2008). En este escenario, la toma de decisiones está en manos del donante y la responsabilidad en el receptor. Es un modelo caracterizado por el establecimiento de relaciones de cooperación asimétricas soportadas en un alto porcentaje en la financiación de proyectos de corto plazo a través de subvenciones a ONG (Martínez y Sanahuja, 2009).

En consecuencia, y como bien lo señalan Sanahuja y Martínez (2009), este enfoque puede ser explicado claramente desde la cadena de la ayuda en la cual se establecen relaciones de tipo vertical alrededor de la financiación y la transferencia de recursos Norte-Sur. Así, los eslabones de la cadena corresponden a los actores que intervienen y las relaciones de poder están representadas de manera descendente. Por lo tanto, en el extremo superior se ubican los donantes quienes toman las decisiones y en el inferior los receptores de la ayuda quienes se convierten en responsables, dependientes de los recursos y subalternos de la cadena. Por su parte, los eslabones intermedios conforman la "industria de la ayuda", junto a la que se encuentran las ONG (Martínez, 2007). 
Un claro ejemplo del modelo anteriormente descrito se puede evidenciar en la cooperación oficial descentralizada de España e Italia articulada desde sus inicios mediante intermediarios, en especial, a través de ONG de desarrollo. La presencia y participación de los gobiernos territoriales es limitada y poco estratégica. Su participación se reduce a los recursos que transfieren a través de convocatorias públicas a organizaciones de la sociedad civil quienes a su vez son las que definen el ámbito de actuación y de ejecución de los proyectos. (Fernández de Losada, 2010)

Esta dinámica comienza a evolucionar con la vinculación de criterios de elegibilidad y de aspectos como la gobernabilidad y el desarrollo local. Sin embargo, esta evolución no esclarece si la participación del gobierno territorial en actividades de cooperación responde a una estrategia de proyección exterior como una acción "natural" o por el contrario, busca seguir reforzando el trabajo asociativo y de las organizaciones de la sociedad civil. (Fernández de Losada, 2010)

Al igual que en la cooperación internacional clásica, bilateral o multilateral, se avanza hacia una opción paralela que prevé una mayor integración entre los actores involucrados en el proceso, esto es, una mayor sinergia en los esfuerzo, complementariedad en la concepción, compromiso y responsabilidad compartida en la ejecución y posterior evaluación. Se resalta, el hecho que implica alejarse de la visión dicotómica y asimétrica entre "donantes" y "beneficiarios", (Malé, 2008) para establecer un patrón de relaciones en las cuales los gobiernos locales están reconocidos como sujetos activos de la cooperación.

Es de esta forma en que surge una relación horizontal, donde cada parte ve a la otra como un socio y en el que las necesidades reales a satisfacer son producto de un consenso previo, previsto y necesario, desde el cual se diseñan las mejores estrategias de beneficio mutuo.

El enfoque horizontal de COD sobrepasa entonces, la diversidad de relaciones verticales soportadas tanto en las subvenciones a ONG como en las relaciones directas entre gobiernos territoriales y avanza hacia una cooperación descentralizada pública basada en relaciones entre iguales que buscan intercambiar experiencias y apoyar las políticas públicas locales y el fortalecimiento institucional.

Estas relaciones de cooperación han avanzado, aunque de manera muy lenta, de esa visión tradicionalista de tinte vertical y asistencialista (enfoque vertical), a una visión que favorece el establecimiento de relaciones más equilibradas basadas en la reciprocidad y el mutuo interés y en formas de actuación más abiertas que fortalecen la acción exterior de los gobiernos locales, y que para muchos debe ser categorizada bajo el "modelo horizontal".

Sin embargo, frente a este último modelo surge una reflexión interesante. De acuerdo con Martínez y Sanahuja (2009), la naturaleza del sistema de ayuda se caracteriza por su arbitrariedad y asimetría por lo que necesariamente se desarrollan relaciones verticales que no permiten avanzar hacia relaciones plenamente horizontales. 
No obstante ello, como en las relaciones de COD pueden diferenciarse distintos niveles de verticalidad, es posible distinguir un tipo ideal de cooperación que comparte algunos elementos del modelo vertical (Martínez y Sanahuja, 2009).

Malé (2008) plantea diferentes modalidades de relaciones de COD entre la Unión Europea y América Latina, que permiten ilustrar tanto las coincidencias y los elementos compartidos entre los dos modelos descritos, como la evolución de la COD hacia un modelo de relaciones más horizontales y reciprocas:

Frente a las intervenciones en ayuda asistencial y humanitaria con énfasis en las situaciones de pobreza y emergencia, plantea una modalidad inicial de relaciones de cooperación indirectas a través de ONG, en las cuales el gobierno local del Norte se convierte en un simple financiador y por tanto, se sustituye por agentes privados. Esta modalidad evoluciona con relaciones de cooperación directa que luego pueden ser materializadas a través de hermanamientos. A su vez, estos hermanamientos permiten movilizar tanto las capacidades institucionales como los esfuerzos solidarios de diversos actores locales, que evidencian una nueva relación de sociedad local a sociedad local. Por último, en muchas ocasiones estas prácticas avanzan hacia la conformación de redes de ciudades hermandadas con el municipio del Sur o viceversa.

Un segundo ejemplo está relacionado con el apoyo a las políticas públicas locales y el fortalecimiento institucional. La evolución en las modalidades de gestión en este eje comienza con relaciones bilaterales verticales soportadas en el apoyo material a la institución del Sur a través de la dotación de materiales, equipamientos, edificios, etc.

Esta modalidad evoluciona cuando se establecen relaciones bilaterales verticales mediante la transferencia de conocimiento, know-how, asistencia técnica, capacitación, asesoramiento del gobierno local del Norte hacia el gobierno local del Sur. Por último, se avanza hacia un tipo de relación bilateral horizontal soportada en el intercambio y en la reciprocidad, que desemboca en redes de gobiernos locales.

Otra manera de superar esa visión asimétrica en el sistema de ayuda Norte-Sur es mediante el estímulo de los vínculos de cooperación descentralizada Sur-Sur para avanzar hacia una mayor horizontalidad y alcanzar las metas y objetivos prioritarios en los planes locales de desarrollo. Frente a esto, muchos municipios en América Latina y el Caribe han emprendido estrategias de cooperación con el fin de fortalecer sus procesos de desarrollo local.

A su vez, la cooperación triangular puede contribuir con este mismo propósito, potencializando la cooperación técnica, a través de la asociación de una fuente tradicional de carácter bilateral o multilateral con un gobierno territorial de desarrollo medio para asistir conjuntamente a un tercer gobierno territorial de menor o similar desarrollo relativo, con proyectos de asistencia técnica e intercambio de buenas prácticas en áreas como el fortalecimiento institucional, la salud, la educación, etc. 
Así por ejemplo, el Programa Urbal de la Unión Europea ha venido estimulando relaciones SurSur mediante experiencias de desarrollo económico local en América Latina. De la misma manera, la promoción de las Agencias de Desarrollo Económico Local (ADEL), promovidas por el Programa Art-Gold, contribuye significativamente con este propósito. Estas experiencias han reforzado la "cooperación inducida", entendida como las acciones de cooperación adelantadas por gobiernos locales en el marco de programas provenientes de organismos multilaterales (Fernández de Losada, 2010).

La experiencia demuestra la diversidad de iniciativas que se vienen promoviendo desde los organismos multilaterales y los espacios de integración y cooperación regional y subregional en materia de cooperación Sur-Sur y cooperación triangular ${ }^{4}$, que sin duda sirven de soporte para impulsar la cooperación descentralizada Sur-Sur y por tanto, una mayor horizontalidad.

\section{LA DINÀMICA DE LA COOPERACIÓN OFICIAL DESCENTRALIZADA EN COLOMBIA: EL CASO DE LAS CC AA EN EL PAÍS (1995-2008)}

En Colombia, el tema de la COD se ha venido promoviendo en diferentes espacios tanto académicos como gubernamentales y de gestión pública local. (Olaya y Velez, 2009). La inclusión de esta modalidad en los gobiernos territoriales ha estado ligada, en la mayoría de los casos, a las estrategias de internacionalización mediante relaciones de cooperación internacional.

Los gobiernos territoriales que han avanzado en la promoción de la internacionalización en los planes de desarrollo departamental y municipal, han incorporado esta modalidad como una estrategia de gestión del desarrollo. Otros, con menor experiencia, han preferido conservar una visión tradicional de la cooperación (Olaya y Velez, 2009).

El estudio de la COD en Colombia, aunque resulta difícil en razón a la ausencia de información consolidada, puede focalizarse, de manera especial, en el caso de las administraciones descentralizadas españolas, principales donantes de este tipo de cooperación en el país. El caso mas representativo está relacionado con la experiencia de las CC AA. La cooperación de estas administraciones autonómicas españolas ha venido adquiriendo un papel cada vez más importante en el sistema de cooperación español (Intermon Oxfam, 2010).

En Colombia, las aportaciones de estas Comunidades se remontan aproximadamente a 1994 y han tenido un crecimiento importante especialmente a partir del año 2000. En la actualidad, el país es considerado como prioritario para 10 de ellas (Madrid, País Vasco, Asturias, Cataluña,

4. Esta fue una de las conclusiones del Propuestas Taller sobre Cooperación Sur-Sur, organizado por el SELA y la Secretaría de Relaciones de México y desarrollado en México el 28 y 29 de julio de 2011. 
Islas Baleares, Castilla y León, Castilla-La Mancha, Cantabria, Valencia y Navarra; para Aragón y la Rioja, Colombia se considera como prioritaria bajo condiciones sectoriales preferentes). Sin embargo, sólo cuatro de ellas (Madrid, Valencia, País Vasco y Cataluña) pueden clasificarse como las de mayor presencia en el país.

Esta es una cooperación que aún no ha sido aprovechada en el país y que apenas se empieza a conocer desde los gobiernos territoriales; a pesar de la dispersión, poca coordinación y articulación de las iniciativas.

Varias son los aspectos que se pueden destacar de la cooperación de estas comunidades en el país entre 1995 y 2007:

En primer lugar, y desde una perspectiva de tiempo, las CC AA con mayor presencia e iniciativas de cooperación al desarrollo han sido Valencia, Madrid, País Vasco y Cataluña.

En este mismo sentido, otras CC AA iniciaron sus actividades de cooperación para el desarrollo a partir del año 2003, aproximadamente, y han adquirido un peso importante dentro del conjunto de la cooperación autonómica española en Colombia. Es el caso de la Comunidades de Islas Baleares, Navarra y Asturias.

Las actividades de Castilla la Mancha comienzan a adquirir una importancia relativa a partir del año 2006, fecha en la que se registran 6 proyectos de cooperación. En esta misma dirección otras comunidades ejecutaron proyectos en el país entre el 2006 y 2008, como es el caso de Aragón (6 proyectos), la Rioja (6 proyectos), Murcia (3 proyectos), Castilla y León (4 proyectos), Extremadura (4 proyectos), Andalucía (4 proyectos) y Cantabria (2 proyectos).

En segundo lugar, la orientación geográfica de cada una de las comunidades evidencia una concentración de la ayuda en tres o cuatro departamentos. País Vasco coopera especialmente en departamentos como Antioquia, Bogotá y Chocó; Valencia en Antioquia, Atlántico, Bogotá y Bolívar; Madrid en Antioquia, Bolívar, Valle del Cauca, Bogotá y Cundinamarca; Cataluña en Bogotá, Valle del Cauca y Bolívar, e Islas Baleares en Santander, Cauca y Antioquia.

Esta concentración responde en la mayoría de los casos a los vínculos de las ONG colombianas con sus pares españolas cuando se trata de subvenciones a proyectos. En otros casos, tiene que ver con la capacidad de gestión internacional de algunos municipios y departamentos colombianos y/o a las relaciones espontáneas que Surgen entre estos y algunos gobiernos autonómicos.

Así por ejemplo, el departamento de Antioquia recibió cooperación de seis de las siete CC AA, con un monto histórico total de 5`619.012€. que equivale al 10\% del total del presupuesto de las comunidades. A su vez, hay una larga lista de municipios que no han recibido COD de 
ninguna comunidad. (Tabla No. 1). Son los casos de Vaupés, Vichada, Tolima, Sucre, San Andrés, Risaralda, Putumayo, Meta, Magdalena, Huila, Guaviare, Córdoba, Guainía y Cesar.

Tabla No. 1 Financiación de la Cooperación

de siete CC AA en Colombia 1995-2008 (euros)

\begin{tabular}{|l|r|r|c|}
\hline $\begin{array}{c}\text { Comunidad } \\
\text { Autónoma }\end{array}$ & \multicolumn{1}{c|}{$\begin{array}{c}\text { Total Euros } \\
\text { donados }\end{array}$} & $\begin{array}{c}\text { Euros donados a } \\
\text { Antioquia }\end{array}$ & $\begin{array}{c}\text { \% frente al total de } \\
\text { euros donados }\end{array}$ \\
\hline País Vasco & $13.270 .592,66$ & 2177059,79 & 16,4 \\
Valencia & $22.677 .099,72$ & 1396985,36 & 6,2 \\
Madrid & 9.081 .844 & 583251 & 6,4 \\
Cataluña & 5.884 .064 & 168000 & 2,9 \\
Asturias & 1.607 .115 & 0 & 0,0 \\
Baleares & $1.384 .015,36$ & 258416 & 18,7 \\
Navarra & 1.676 .023 & 1035300 & 61,8 \\
Total & $55.580 .753,74 €$ & $5.619 .012,15 €$ & 10,1 \\
\hline
\end{tabular}

Fuente: Elaboración propia, a partir de datos suministrados por AECID, Colombia y de algunas CC AA

Continuando con el comportamiento de la orientación geográfica y la financiación de la cooperación, es posible observar que departamentos como Bolívar han sido foco de COD; En el periodo de 1995 a 2006 el departamento de Bolívar recibió aproximadamente el 12\% del total de euros financiados por las comunidades españolas en toda Colombia.

Por su parte la cooperación de estas siete CC AA destinó a Bogotá niveles similares de financiación de los departamentos mencionados (Antioquia y Bolívar). La gran diferencia es que una sola ciudad recibió el $8.6 \%$ del total de euros financiados. Bogotá ha concentrado una importante muestra de iniciativas de cooperación autonómica; aunque esto no parece negativo, lo que revela es que esta ciudad relegó del mapa al departamento de Cundinamarca, que recibió aproximadamente el 1,17\% del total de euros donados entre 1995 y 2006.

En síntesis, lo que indica este análisis inicial es que en Colombia tan solo cuatro de las 17 CC AA han tenido una presencia significativa en el país, que a su vez permite observar que si bien, es una cooperación que cada vez adquiere más fuerza en el país, no siempre responde a las necesidades más próximas locales y por el contrario, se focaliza en aquellos municipios o departamentos caracterizados por altos niveles de desarrollo y de capacidad de gestión. 
En tercer lugar, las siete CC AA, sin excepción, han volcado su cooperación al sector de infraestructuras sociales. Así entonces, del total de la financiación de estas comunidades entre 1995 y $2008^{5}$, aproximadamente el $68 \%$, ha sido invertido en proyectos clasificados en este sector de actividad

De manera detallada, la siguiente tabla permite observar el número de proyectos que justifican este porcentaje en infraestructuras sociales, frente al total de proyectos registrados.

Tabla No. 2 Cooperación de siete CC AA en Colombia en el sector infraestructuras sociales 1995-2008

\begin{tabular}{|l|c|c|c|}
\hline $\begin{array}{c}\text { Comunidad } \\
\text { Autónoma }\end{array}$ & $\begin{array}{c}\text { No. de proyectos } \\
\text { infraestructuras } \\
\text { sociales }\end{array}$ & $\begin{array}{c}\text { Total proyectos } \\
\text { registrados } \\
\text { por Comunidad }\end{array}$ & $\begin{array}{c}\text { \% del total } \\
\text { donado }\end{array}$ \\
\hline País Vasco & 84 & 119 & 70,5 \\
Valencia & 103 & 159 & 64,7 \\
Madrid & 65 & 99 & 65,6 \\
Catalunya & 62 & 84 & 73,8 \\
Asturias & 17 & 32 & 53,1 \\
Baleares & 25 & 39 & 64,1 \\
Navarra & 34 & 43 & 79 \\
Total & $\mathbf{3 9 0}$ & $\mathbf{5 7 5}$ & $68 \%$ apróx. \\
\hline
\end{tabular}

Fuente: Elaboración propia, a partir de datos suministrados por AECID, Colombia y algunas CC AA

En cuarto lugar, y desde la perspectiva de las organizaciones con las que han trabajado estas CC AA en Colombia, se observa que un gran porcentaje de la cooperación autonómica se realiza a través de subvenciones a proyectos presentados por ONG.

Las ONG son las organizaciones que más euros reciben de las CC AA, estas son las instituciones por excelencia de la COD así como lo son en menor medida las fundaciones que para el caso de Madrid se ubican en primer lugar en términos de financiación. Pese a esta concentración de la ayuda en manos de las ONG, que se soporta en relaciones de tipo vertical y asistencialista,

5. Se incluyen algunos proyectos del 2009. 
desde el año 2008 (apróx), se promueve la presencia de otros actores, que aunque de manera muy lenta, dan muestra de la apertura en las modalidades y modos de gestión. Así por ejemplo, se empiezan a evidenciar los aportes de las CC AA a organismos internacionales y relaciones de cooperación directa con gobiernos no centrales colombianos, con entidades gubernamentales del nivel nacional y organizaciones de la sociedad civil.

Tabla No. 3 Algunos actores con los que han trabajado las principales CC AA presentes en Colombia (2004-2008)

\begin{tabular}{|c|c|c|}
\hline $\begin{array}{l}\text { Comunidad } \\
\text { Autónoma }\end{array}$ & $\begin{array}{c}\text { ONG frente a demás instituciones } \\
\text { en /comunidad }\end{array}$ & Otros actores $^{6}$ \\
\hline País Vasco & Primera institución más financiada & PNUD, OCHA, alcaldías \\
\hline Valencia & Primera institución más financiada & PNUD, ACNUR \\
\hline Madrid & Segunda institución más financiada & $\begin{array}{l}\text { Fundaciones colombianas, Ministerios, } \\
\text { Acción Social, Presidencia. }\end{array}$ \\
\hline Cataluña & Primera institución más financiada & $\begin{array}{l}\text { PNUD, Alcaldías, gobernaciones, } \\
\text { Procuraduría }\end{array}$ \\
\hline Asturias & Primera institución más financiada & ACNUR, alcaldías \\
\hline Baleares & Primera institución más financiada & Alcaldías \\
\hline Navarra & Primera institución más financiada & No registra \\
\hline Asturias & Primera institución más financiada & ACNUR, alcaldías \\
\hline Andalucía & Primera institución más financiada & OEI, Alcaldías \\
\hline
\end{tabular}

Fuente: Elaboración propia a partir de datos suministrados por AECID y algunas CC AA

Esta primera contextualización de la presencia de las CC AA en el país, permite identificar algunos puntos débiles que justifican la necesidad de avanzar hacia dinámicas más incluyentes, reciprocas y horizontales de COD que beneficien los procesos de desarrollo territorial en Colombia:

\section{Desde los gobiernos autonómicos españoles}

Si bien, las CC AA avanzan cada vez más en la institucionalización de su cooperación a través de planes, políticas y mecanismos de planificación y coordinación, aún se observa una brecha entre estos y las prioridades del país.

6. Datos aproximados suministrados por AECID (Colombia). 
En primer lugar, se evidencian claros problemas de información y dispersión. Los datos de la cooperación autonómica en el país son difíciles de conocer, debido a que no existen fuentes consolidadas que registren en su totalidad los proyectos ejecutados y el impacto de estos. Paradójicamente, la fuente más cercana es AECID (Colombia), que maneja algunas bases de datos del $70 \%$ de los proyectos ejecutados en el país.

Bajo esta misma lógica, los mecanismos de seguimiento, evaluación e impacto de los proyectos ejecutados por las ONG, se reducen en la mayoría de los casos a justificaciones técnicas y económicas intermedias y finales.

Ligado a lo anterior, se observa una ausencia generalizada y una falta de implicación directa del gobierno autonómico, que actúa como un simple financiador, reforzando así, el modelo clásico y vertical de la ayuda inducido por la demanda sin un norte estratégico común. Adicionalmente, la falta de implicación del gobierno local del país, no genera ningún tipo de coordinación y articulación con las estrategias de desarrollo local ni contribuye con los procesos de alineación, planificación y aprendizaje entre ambas partes.

De la misma manera, y como consecuencia de esa lógica asistencialista y vertical, las relaciones de cooperación se limitan a las prioridades de los gobiernos autonómicos, que se materializan nuevamente a través de los proyectos financiados a las ONG.

Se infiere entonces, que en la mayoría de los casos no existen planes conjuntos y planificados entre los agentes españoles y colombianos. Con esto no se logran establecer objetivos claros y compartidos, ni prioridades sectoriales y necesidades concertadas.

La dinámica también revela que este modelo vertical responde, entre otras cosas, a la misma concepción asumida por los gobiernos locales y regionales colombianos. En palabras de Martinez y Sanahuja (2009), "el modelo vertical de cooperación descentralizada es una consecuencia de la propia actitud de los gobiernos descentralizados del Sur, que conciben a los gobiernos descentralizados del Norte exclusivamente como financiadores a través de los cuales obtener recursos".

Este escenario plantea una discusión adicional que permite identificar algunos de los problemas relacionados con los procesos de cooperación de los gobiernos territoriales colombianos.

\section{Desde los gobiernos territoriales colombianos}

En términos generales, en el país se evidencia un desconocimiento extendido por parte de muchas de las administraciones territoriales referente a los diversos mecanismos o modalidades de internacionalización y dentro de estas, la COD. De lo anterior se desprende la limitada 
participación de estas administraciones en procesos o practicas de COD tendientes a fortalecer la gestión pública y los procesos de desarrollo local'

Son muy pocos los municipios o ciudades en Colombia que cuentan con una espacio en el cual se trabajan los asuntos internacionales. De acuerdo con el Informe Experiencias en internacionalización municipal en Colombia (FCM-UJTL, 2008), únicamente en algunas gobernaciones y ciudades capitales como Medellín, Bogotá, Bucaramanga, Cartagena, Manizales, Pereira, Pasto, Cali y Florencia, existen espacios de gestión de asuntos internacionales establecidas en la estructura administrativa de las Alcaldías; por lo demás, en la mayoría de los casos, las responsabilidades sobre los proyectos que tienen relación con el tema internacional, recaen en las oficinas de planeación, en el despacho del Alcalde o en algún asesor externo.

Se destaca que en el caso de Medellín, existe una institucionalidad más avanzada a través de la creación de una agencia propia, Agencia de Cooperación e Inversión de Medellín y el Área Metropolitana, que tiene el objetivo de propiciar el desarrollo de la ciudad a través de la creación de relaciones con otros países y ciudades.

Sin embargo, existen casos destacables por su excepcionalidad, ya que, en ausencia de una oficina de asuntos internacionales, la motivación y voluntad política de líderes han logrado avances significativos hacia el posicionamiento de sus municipios en la escena internacional. Un ejemplo de ello es el municipio de Caldono en el Departamento del Cauca, que producto de las gestiones de su Alcaldesa (2008-2011) Enelia Salinas Chivatá, llevó el nombre de su municipio a instancias como la Embajada del Japón, la Unión Iberoamericana de Municipalistas, IKVPAX CHRISTI de Holanda, la Universidad de Lleida, la Agencia Asturiana de Cooperación, entre otras.

Aunque esto demuestra que algunas ciudades/municipios del país tienen experiencia en actividades del ámbito exterior, en la mayoría de los casos, no se cuenta con políticas o estructuras sostenibles de cooperación internacional, que permitan vincular la dimensión internacional en lo local de una manera sostenible y eficaz. Ante esto y debido a la naturaleza esporádica de las prácticas se evidencia una ausencia de planificación y de marcos estratégicos que limita considerablemente el alcance de las mismas y que nuevamente refuerza el modelo vertical de cooperación.

Así entonces, en la mayoría de los casos las actividades específicamente asociadas a la cooperación descentralizada de los municipios, al igual que el resto de las actividades de internacionali-

7. Esta fue una de las conclusiones de la investigación adelantada por el Programa de Relaciones Internacionales en convenio con la Federación Colombiana de Municipios en diciembre de 2007 sobre los procesos de internacionalización municipal en el país. 
zación, no se adelanta en forma planificada y sostenida, a partir de estrategias o políticas de mediano y largo plazo, sino que surgen de necesidades específicas o de acuerdos espontáneos con otros municipios nacionales o extranjeros. Por otra parte, son los municipios más grandes, los que de igual manera, logran un mayor nivel de desarrollo en los temas de cooperación descentralizada.

Bajo este mismo escenario y como consecuencia de esto, es común identificar que estas actividades de los gobiernos territoriales colombianos mantienen el enfoque vertical y asistencialista a través de la financiación de proyectos, con especial énfasis en las prioridades de los "donantes".

De la misma manera, y ligado a estas dificultades, los gobiernos territoriales colombianos se ha enfrentado a una tendencia centralizadora de la cooperación internacional desde las instancias gubernamentales coordinadoras que en algunos casos, no han generado articulación entre las prioridades territoriales y del orden nacional. Aun así, algunos gobiernos locales han ganado espacios en el desarrollo de capacidades de gestión autónoma que les ha permitido establecer procesos de apropiación local proyectables al exterior.

Por último, si bien algunos municipios del país han adelantando iniciativas de cooperación descentralizada, las limitaciones para establecer vínculos de este tipo obedecen a la falta de desarrollo de capacidades, al vago entendimiento de lo que significa y al desconocimiento por parte de muchas de las administraciones locales de menor desarrollo referente a los diversos mecanismos o modalidades dirigidos a los gobiernos municipales.

\section{CONCLUSIONES}

El panorama de la cooperación descentralizada en el país sigue mostrando un evidente problema de enfoque, información, capacitación, gestión y claridad de lo que significa. Con esto, se desaprovechan los recursos y los mecanismos para establecer vínculos de cooperación entre homólogos y se desarrollan iniciativas dispersas con escasa capacidad transformadora.

No obstante los problemas evidenciados, se puede percibir un creciente interés por parte de muchas administraciones locales para establecer vínculos de cooperación descentralizada a través de mecanismos planificados de gestión y marcos de acción que contribuyan con procesos más horizontales y equitativos y con el fortalecimiento institucional, local y participativo.

Así entonces, la experiencia significativa de la cooperación de las CC AA en el país, evidencia la necesidad de establecer nuevas dinámicas y mecanismos para evolucionar de la concepción clásica, dominante y asistencialista que alimenta "la cadena de la ayuda" a un enfoque basado en relaciones estratégicas, de largo plazo, que pasen de la financiación de proyectos a través de 
las ONG a unas relaciones más duraderas entre socios, y que contribuyan con los procesos de descentralización, fortalecimiento institucional y gobernanza local.

Bajo este escenario, es posible señalar algunos elementos de reflexión que contribuyen con este propósito:

\section{Desde los gobiernos autonómicos:}

- $\quad$ Avanzar en procesos de cooperación directa orientado al apoyo de las políticas públicas y al fortalecimiento institucional.

- Cambiar el enfoque reduccionista de financiación de proyectos a las ONG, diversificando y fortaleciendo los actores, instrumentos y modos de gestión que favorezcan la alineación y la planificación estratégica conjunta.

- Avanzar en las relaciones multiactor y multinivel y promover el esquema de redes

\section{Desde los gobiernos locales colombianos:}

- Ampliar el campo de autonomía y de liderazgo en la cooperación, desde los municipios colombianos.

- Generen capacidades en la sociedad civil organizada para afianzar su participación y compromiso en las iniciativas públicas alrededor de la internacionalización, en especial, la cooperación internacional. La gestión desde abajo hacia arriba constituye la clave para el impulso y sostenimiento de una estrategia de relacionamiento horizontal de cooperación descentralizada con enfoque participativo.

- Vincular en los Planes de Desarrollo Municipal la cooperación internacional y/o la internacionalización como estrategia para la consecución de fondos, el establecimiento de relaciones internacionales, el fortalecimiento de capacidades institucionales y la ejecución de proyectos.

- $\quad$ Establecer estructuras y procedimientos organizativos para gestionar las relaciones de cooperación. (a través de una secretaría independiente, la Secretaría de Hacienda, de Planeación o de Desarrollo Económico, del despacho del Alcalde, de unidad de asesoría y apoyo o unidad técnica especializada, de un comité interinstitucional, de asociaciones de municipios y/o las Agencias de Desarrollo Económico Local (ADEL) o a través de la creación de una agencia independiente. 
- Identificar características, potencialidades y necesidades del municipio, proyectables al exterior en conjunto con la sociedad civil.

- Identificar socios, modalidades e instrumentos de cooperación oficial descentralizada que avancen hacia relaciones más horizontales y de interés común. Bajo esta perspectiva, el panorama de la COD muestra diferentes actores que contribuyen con este propósito como gobiernos locales en América Latina, programas de cooperación al interior de los organismos de integración regional, programas de cooperación Sur-Sur y triangular, promovidos desde actores multilaterales y bilaterales de cooperación (Art-Gold de PNUD, Urbal de la Unión Europea, la Agencia Japonesa de Cooperación Internacional (JICA), la Agencia Alemana de Cooperación (GIZ), entre otros), redes de gobiernos locales (CGLU, FLACMA, OLAGI, RAC, UIM etc),

- Construir estrategias de cooperación desde las entidades territoriales colombianas con la participación de la sociedad civil.

- Desarrollar una agenda de acción exterior para establecer relaciones de cooperación de acuerdo con las prioridades municipales

- Generar espacios de planificación conjunta entre los actores de COD, que vinculen la identificación de las prioridades locales y su articulación con los planes rectores de cooperación de los pares internacionales (objetivos compartidos entre pares internacionales y entre pares internacionales y beneficiarios).

- Avanzar hacia el establecimiento de una política pública de internacionalización y/o cooperación internacional que vinculen la COD.

Sin duda alguna, el reto para los municipios en Colombia consiste en desarrollar capacidades y estrategias que orienten una agenda de acción exterior de cooperación descentralizada en el marco de una política circunscrita en la agenda pública local, inscrita en el contexto propio y estratégico de la acción pública impulsada desde la administración local. (Sanz, 2008).

Para esto, los procesos de cooperación descentralizada deben estar soportados en la conciencia de los gobiernos locales de su territorio como base fundamental para comprender la dinámica política, económica y social y como el elemento diferenciador desde el que se construye la identidad colectiva e individual.

Ligado a esto, lo fundamental es construir capacidades y generar un cambio de enfoque desde los municipios colombianos en el cual se abandone la tradicional formula "donante-receptor" $y$ se avance hacia el concepto de "socios", que se interesen por ejecutar proyectos de mutuo interés, intercambiar conocimientos, buenas prácticas, en beneficio del desarrollo local. 


\section{BIBLIOGRAFÍA}

- $\quad$ Aguirre, Z.I. (2000). ¿Qué sentido tiene hablar de paradiplomacia? Una encuesta intertextual en torno a un neologismo polisémico? En Aldecoa, F., y Keating, M. (dirs.), Paradiplomacia: las relaciones internacionales de las regiones. (pp. 203-235.) Marcial Pons- Ediciones Jurídicas y Sociales, Madrid,

- Alburquerque, F. (2004). Desarrollo económico local y descentralización en América Latina. En: Revista de la CEPAL Nro. 82.

- $\quad$ Aldecoa, Francisco y Keating, Michael. (2000). Paradiplomacia: las relaciones internacionales de las regiones, Madrid, Marcial Pons.

- $\quad$ Alonso, J.A., Garcimartín, C., \& Martin, V. (2010). Ayuda, calidad institucional e imposición: Algunos desafíos para el sistema de cooperación internacional. Conference on Development Cooperation in Times of Crisis and on Achieving the Mdgs. Recuperado de http://www.eu2010.es/export/sites/presidencia/comun/descargas/agenda/agenda_junio/ pdf_grupo/ES/Alonso_xAyudax_calidad_institucional_ex.pdf

- Díaz A, L. (2008). La COD, cambio y resistencia en las relaciones internacionales contemporáneas, Madrid. Catarata.

- $\quad$ Duchacek, Ivo D. (1984). "The international dimension of subnational self-government". Oxford University Press. 14 (4). Federated States and International Relations. Recuperado de www.jstor.org/stable/3330188

- Federación Colombiana de Municipios. FCM. (2009) Guía práctica para la Internacionalización Municipal. Una invitación estratégica iDe cara al mundo! Gestión internacional de gobiernos locales y asociaciones municipales. Recuperado de http://www.fcm.org.co/ fileadmin/gestion/pdf/Fasciculo1.pdf.

- $\quad$ Fernández de Losada, A. (2010). "Revisión de los Fundamentos de la Cooperación Descentralizada Pública Local". Montevideo. Observatorio de Cooperación Descentralizada UE-AL. Recuperado de http://www.observ-ocd.org/temp/libreria-340.pdf

- $\quad$ Foro de Alto Nivel de París. (2005). "Declaración de París sobre la eficacia de la ayuda al desarrollo. Apropiación, armonización, alineación, resultados y mutua responsabilidad", París.

- Intermón Oxfam (2010). La realidad de la ayuda 2009, una evaluación independiente de la ayuda y las políticas de desarrollo en tiempos de crisis. España, Intermón Oxfam. 
- Malé, J.P. (2008). Panorámica de las prácticas y tendencias actuales de la cooperación descentralizada pública. Montevideo, Observatorio de Cooperación Descentralizada UEAL. Recuperado de http://www.observ-ocd.org/temp/libreria-199.pdf.

- Martínez, Ignacio y Sanahuja, José A. (2009). La agenda internacional de eficacia de la ayuda y la cooperación descentralizada de España, Madrid, Fundación Carolina.

- Martínez, I. (2007). La cooperación no gubernamental española en Perú, Madrid. Instituto Complutense de Estudios Internacionales, ICEI. Recuperado de http://eprints.ucm.es/9637/ 1/Estudios_e_informes_n\%C2\%BA_2.pdf

- $\quad$ Olaya, Sandra y Velez, Jeanette. (2009). La cooperación descentralizada en Colombia: una primera aproximación a la visión de los Departamentos y la experiencia de Bogotá como Distrito Capital. Montevideo, Observatorio de Cooperación Descentralizada UE-AL. Recuperado de http://www.observ-ocd.org/temp/libreria-264.pdf

- Pérez, S. (2007). El contexto global y la internacionalización de los municipios en los procesos de desarrollo territorial. Revista OIDLES 1(0). Recuperado de http:// www.eumed.net/rev/oidles/00/Rozzi.htm

- $\quad$ Rodríguez, G. S. (2005, 25 de abril) La Inserción Internacional De Chile. Perspectivas de las regiones. Encuentro Nacional Ciudad De Punta Arenas, Chile. Recuperado de www.cooperaciondescentralizada.Gov.Cl/1511/Articles-341_Recurso 1.Pdf.

- Sanz, C.B. (2008). Guía para la acción exterior de los gobiernos no centrales y la cooperación descentralizada Unión Europea - América Latina, Elementos para la construcción de una política pública local de cooperación descentralizada, En Observatorio de la cooperación descentralizada Unión Europea-América Latina. Vol. 2, Barcelona.

- $\quad$ Sistema de información en Cooperación Descentralizada, SICDE. Universidad Jorge Tadeo Lozano. Programa de Relaciones Internacionales.

- Zapata, G.E. (2007). Manual práctico para internacionalizar la ciudad. Guía para la acción exterior de los gobiernos no centrales y la cooperación descentralizada Unión Europea América Latina, Vol. 1, Barcelona, Observatorio de la cooperación descentralizada Unión Europea-América Latina. 